\title{
EVALUACIÓN DE CONDUCTOS ACCESORIOS DEL MAXILAR SUPERIOR
}

\author{
EVALUATION OF UPPER MAXILLARY ACCESSORY CANALS
}

Natalia Nilda Soto Terán ${ }^{1}$

\section{RESUMEN}

Introducción: En la región anterosuperior, además del conducto nasopalatino, podemos encontrar otros conductos neurovasculares que pueden ser evaluados por medio de tomografía computarizada de haz cónico. El propósito de este estudio es determinar la presencia, número, diámetro, dirección y emergencia de conductos accesorios en áreas dentadas y desdentadas en la población de Cochabamba. Materiales y métodos: Se evaluaron 300 tomografías computarizadas de haz cónico de pacientes que acudieron al centro radiográfico CERPAX, mayores a 15 años y con un tamaño de voxel mínimo de 0,2 mm. Resultados: Se encontró una frecuencia de $47,33 \%$ de conductos accesorios con un diámetro igual 0 mayor a $1 \mathrm{~mm}$. Se halló a un paciente con 4 conductos accesorios. El diámetro medio de los conductos fue de $1,44 \mathrm{~mm}$ y el máximo de 2,80 $\mathrm{mm}$. El diámetro fue mayor en zonas desdentadas. La mayor emergencia se encontró en la región de los incisivos centrales y a nivel de la tabla ósea palatina. Hubo asociación significativa entre la emergencia a nivel de las tablas óseas y el estado dental de la zona. La mayor parte de los conductos tiene dirección hacia el canalis sinuosus. Conclusiones: El diámetro mayor encontrado es el valor máximo hasta la fecha. El diámetro es mayor en áreas desdentadas que en dentadas. Es importante tomar en cuenta a los conductos accesorios de esta región antes de cualquier procedimiento quirúrgico en la zona a fin de evitar una lesión en el paquete vasculonervioso.

Palabras clave: conducto accesorio, canalissinuosus, conductoneurovascular, tomografía computarizada de haz cónico

Citar como: Soto N. Evaluación de conductos accesorios del maxilar superior. Rev Cient Odontol (Lima). 2018; 6 (2): 143-157. 


\section{ABSTRACT}

Introduction: In the anterior superior region, in addition to the nasopalatine duct, we find other neurovascular canals that can be evaluated using cone beam computed tomography. The purpose of this study is to determine the presence, number, diameter, direction and emergence of accessory canals in dentate and edentulous members of the population of Cochabamba. Material and Methods: 300 cone beam computed tomography scans were evaluated, from patients at the CERPAX radiographic center, aged over 15 years and with a minimum voxel size of $0.2 \mathrm{~mm}$. Results: The frequency of accessory canals with a diameter equal to or greater than $1 \mathrm{~mm}$ was $47.33 \%$. One patient was found to have 4 accessory canals. The average diameter of the canals was $1.44 \mathrm{~mm}$ and the maximum diameter was $2.80 \mathrm{~mm}$. The diameter was greater in edentulous areas. The greatest emergence was found in the region of the central incisors and at the level of the palatal bone. There was a significant association between emergence at the level of the bone and the dental condition of the area. Most of the canals were oriented towards the canalis sinuosus. Conclusions: The greatest diameter found is the maximum value to date. The diameter is greater in edentulous areas than in dentate areas. It is important to take into account the accessory canals of this region before engaging in any surgical procedure in the area, in order to avoid damage to the neurovascular bundle.

Keywords: Accessory canal, canalis sinuosus, neurovascular canal, cone beam computed tomography.

\section{INTRODUCCIÓN}

Aunque la estructura anatómica neurovascular más conocida en la región anterior del maxilar superior es el conducto nasopalatino, que podemos identificar en radiografías bidimensionales junto a su foramen incisivo (1), en esta zona también debemos considerar otros conductos óseos que pueden contener elementos neurovasculares y que son difícilmente identificables en radiografías bidimensionales (2, 3). Estos conductos accesorios son relativamente frecuentes y de relevancia clínica, dado que las estructuras neurovasculares que albergan pueden ser de calibre significativo $(2,4)$. Dichos conductos han recibido varias denominaciones: conducto lateral incisivo, variaciones neurovasculares en el paladar anterior y canales accesorios o canales adicionales. Muchos de ellos tienen en común su comunicación con el canalis sinuosus $(\mathrm{CS})(4,5,6)$.

Eshak et al. (7) describen como conducto accesorio a toda línea radiolúcida o hipodensa que no está descrita anteriormente como un hito anatómico en la literatura de la radiología oral, que no está presente en todos los pacientes y que no está relacionada con ningún tipo de patología.

El canalis sinuosus es un conducto óseo por donde recorre el nervio, la arteria y la vena alveolar anterior superior (AAS), que proveen irrigación e inervación a las piezas dentarias anterosuperiores y tejidos blandos adyacentes $(2,8,9)$. El término canalis sinuosus hace referencia al doble recorrido curvo que realiza este conducto que se extiende por unos 55 $\mathrm{mm}$ a través del maxilar (10).

Los dientes superiores son inervados por tres nervios alveolares superiores que nacen del nervio maxilar superior: dentario o alveolar posterior, alveolar medio y alveolar anterior (11). Los 
nervios alveolares superiores posteriores emergen en la fosa pterigopalatina, justo antes de que el nervio infraorbitario ingrese en el extremo posterior del canal infraorbitario (12). El nervio alveolar anterior superior es un solo tronco de considerable tamaño en la mayor parte de su curso, que nace de la cara lateral del canal infraorbitario, cerca de su punto medio $(6,14)$. También puede emerger de la cara inferior del conducto infraorbitario $(13,15)$. Olenczak et al. (16) demostraron por medio de disecciones que el origen era entre 10 y $30 \mathrm{~mm}$ por detrás al borde infraorbitario. Disecciones y cortes histológicos de este conducto revelan la existencia de nervios y vasos sanguíneos (11).

Desde su origen, tiene un trayecto hacia abajo, adelante y medialmente hacia la pared anterior del seno maxilar, en una curva templada que pasa debajo del foramen infraorbitario, hasta llegar al borde anterior de la abertura piriforme, donde cambia de dirección bruscamente hacia abajo para contornear a esta abertura y abrirse delante del tabique nasal, en el foramen septal, que es una estructura bilateral que se encuentra cerca al piso de la espina nasal anterior $(2,6,8$, 12).

Debido a su alta frecuencia, el CS es considerado una estructura anatómica normal que puede ser localizado por medio de estudios de tomografía computarizada de haz cónico (TCHC) $(2,4,5,8$, 14). No obstante, su apertura en la región anterior del maxilar superior puede ser considerado como variante anatómica $(2,3)$. Aunque un gran porcentaje de estos canales o conductos accesorios se continúan con el CS, también pueden originarse en el piso de la cavidad nasal o en el conducto nasopalatino mismo (2). Los llamados agujeros de Scarpa son dos conductos menores del conducto nasopalatino que transmiten los nervios nasopalatinos $(7,17,18)$. Otros autores, consideran que la emergencia del CS está próxima a la espina nasal anterior, de modo que los canales que se encuentran por debajo del piso de las fosas nasales serían los canales accesorios del CS o variaciones anatómicas en el paladar anterior $(2,5,14,19)$.

Desde su nacimiento en el CS, el trayecto de los canales accesorios puede ser palatal, transversal o labial. De acuerdo con esta localización espacial, la emergencia se da por palatino de las piezas dentarias anterosuperiores (curso palatal), en la cima del reborde óseo (transversal) o en la cortical ósea vestibular (labial) (14). La emergencia de estos canales, según de Oliveira et al. (2), siempre es palatina y puede darse en la región de los incisivos centrales, entre los incisivos central y lateral, en la región de los incisivos laterales, en la región canina, en la región del primer premolar, lateral al foramen incisivo, anterior al foramen incisivo y posterior al foramen incisivo.

La estética dental, en los últimos años, ha cobrado mayor importancia, principalmente en el sector anterior, lo que hace necesaria la implementación de técnicas de rehabilitación de piezas dentarias con el uso de implantes dentales, evidenciándose fracasos en los tratamientos y haciendo de la región anterior superior un reto estético para el posicionamiento de implantes, por lo que es una práctica rutinaria $(20,21)$.

Después de las exodoncias de piezas anterosuperiores, puede existir reabsorción ósea alveolar que requiera de procedimientos de injertos óseos en la zona para su posterior rehabilitación con implantes dentales $(5,22)$. Durante alguno de los procedimientos quirúrgicos, pueden ser dañadas estructuras neurovasculares, lo que genera un riesgo de hemorragia y disfunciones sensitivas (hiperestesia, parestesia o dolor) $(1,2$, 
$14,17,20,19,23)$. No obstante, Apostolakis et al. (24) indican que la hemorragia no es un problema clínico real que represente riesgo potencial para el paciente por el limitado diámetro de los vasos sanguíneos alveolares superiores, y es fácilmente controlable en pacientes con hemostasia normal por dentistas experimentados, de modo que el riesgo es el sangrado durante la cirugía que genere el deterioro de la visión del campo quirúrgico. También se da la hipótesis de que dicho daño afecte la fase postoperatoria impidiendo la oseointegración de implantes dentales en la zona $(14,17,20,21,22)$ y la falta de irrigación del injerto óseo $(23,24)$. Se han reportado casos con un diámetro de dicho conducto de 2,58 mm (14).

Machado et al. (14) grafican el caso de un implante dental en relación con el canal accesorio del CS con dolor posperatorio que se alivia después de retirar el implante en la zona. El desconocimiento de esa estructura puede llevar a errores en el diagnóstico, al ser confundida con otras estructuras anatómicas o lesiones. Shelley et al. (22) describen el caso de una radiolucidez periapical proyectada en la zona apical de un canino superior en una radiografía periapical que simula una lesión inflamatoria periapical, otras proyecciones revelan bordes corticalizados típicos de conductos neurovasculares. También pueden ser malinterpretados como líneas de fractura (7).

Muchos estudios e investigaciones anteriores de los conductos óseos, principalmente aquellos de calibre menor, consisten en disecciones macroanatómicas de cadáveres humanos. Murakami et al. (25), en 1994, realizaron un estudio de la distribución de los nervios alveolares superiores anterior, medio y posterior en 37 cadáveres utilizando impregnación de plata para poder evaluar tanto el recorrido intraóseo (por conductos o canales) como el extraóseo (por la mucosa sinusal). La resonancia magnética de alta resolución fue usada en la investigación para estudiar la microanatomía sin necesidad de realizar cortes histológicos (18).

La tomografía computarizada de haz cónico (TCHC) ha generado mayor confianza por parte del clínico en la imagen radiográfica, ya que estos canales neurovasculares de calibre reducido no pueden ser observados adecuadamente por medio de las radiografías bidimensionales $(8,26)$. Permite una mejor evaluación preoperatoria de la zona, un diagnóstico más preciso y, por ende, una planificación más adecuada del tratamiento, mostrando la ubicación exacta de los conductos neurovasculares, su diámetro y proximidad con otras estructuras (26). La TCHC es el estudio ideal para la evaluación de conductos accesorios, dado que permite ajustar los campos de visión, por la calidad y precisión para la evaluación de detalles, elimina las imágenes superpuestas, permite recoger mediciones lineales o angulares confiables y la reconstrucción tridimensional (9). Es importante resaltar que el tamaño de voxel con el que se obtenga la imagen tomográfica juega un papel de suma importancia en la identificación de estos conductos accesorios, dado que el tamaño de voxel debe ser la mitad del objeto representado para evitar el efecto de volumen parcial que podría desvanecer la imagen del canal. De modo que, para que sean representados canales de $0,5 \mathrm{~mm}$ de diámetro, como indica la literatura, debemos tener un tamaño de voxel de 0,25 mm. Además del tamaño de voxel, es importante la resolución de contraste (capacidad de diferenciar dos objetos con alto contraste que estén unidos), el ruido y los artefactos (24). Los movimientos menores del paciente durante la adquisición de la imagen generan imprecisión en las corticales óseas de los conductos (27). 
La pérdida de piezas dentarias en los maxilares da lugar a la atrofia alveolar en un proceso complejo y multifactorial donde intervienen factores mecánicos, hormonales, nutricionales, metabólicos, ambientales, socioeconómicos y características étnicas; no obstante, dado que la reabsorción alveolar ocurre aun cuando el estado óseo del resto del cuerpo es bueno, los factores funcionales locales juegan un rol importante, pues la reabsorción del hueso residual en zonas edéntulas es un proceso individual que no puede predecirse con precisión. El estado dental del paciente juega un rol mucho más importante en la anatomía ósea que el género del paciente (28). El edentulismo altera la función masticatoria y la ingesta alimenticia, lo que modifica los patrones de estrés biomecánico que, a su vez, influyen en la morfología de los maxilares afectando la remodelación de hueso (29). La altura alveolar de mandíbulas edéntulas es menor que en las dentadas, el reborde alveolar es de menor tamaño, tanto en el sentido horizontal como en el vertical y en el ancho por la atrofia de desuso y la reabsorción ósea alveolar $(21,29)$. La pérdida de las piezas dentarias genera reabsorción de la cresta ósea alveolar de manera progresiva, de modo que la cresta ósea se aproxima a estructuras anatómicas como el conducto nasopalatino y otros canales vasculares, lo que da como resultado la emergencia de los mismos en la cima de la cresta ósea por la migración del alveolo hacia el palatino.

Mardinger et al. (27), en su investigación respecto los cambios morfológicos del conducto nasopalatino con la ausencia de piezas dentarias, encontraron que el diámetro del conducto es mayor mientras mayor sea el grado de reabsorción de la cresta alveolar, indicando que la abertura palatal, en casos en los que se encuentra situada en la cima del reborde por reabsorción ósea, puede llegar a ocupar el 35,7\% del área destinada al implante dental. El conducto nasopalatino, al igual que muchos conductos, no son estructuras estáticas en el tiempo, sino que se agrandan con la edad y con la ausencia de dientes. Este proceso es similar al que ocurre con la expansión del seno maxilar después de la pérdida de las piezas dentarias adyacentes (27). Ozcakir et al. (1) y Tozum et al. (30) no encontraron diferencias significativas en el diámetro del conducto nasopalatino en pacientes con y sin dientes anteriores.

Existen 9 estudios similares al presente, realizados entre los años 2010 y 2017 en Bélgica (23, 2), Suiza (5), Turquía $(4,19)$, México (7) y Brasil $(8,9,14)$.

El objetivo del presente estudio es el de determinar la presencia, número, diámetro y emergencia de los conductos accesorios de la región anterior del maxilar superior en zonas dentadas y desdentadas, mediante la evaluación tomográfica. Por ello, arroja nuevos conocimientos sobre la frecuencia y calibre de estos conductos en la población cochabambina, dado que existen muy pocos estudios en contextos poblacionales diferentes. También aporta datos sobre la emergencia de estos conductos en un análisis comparativo de zonas dentadas y desdentadas, investigación no presente en la literatura, siendo de carácter innovador.

\section{MATERIALES Y MÉTODOS}

El presente estudio es de carácter transversal, retrospectivo, descriptivo y comparativo. La muestra es de 300 tomografías computarizadas de haz cónico de pacientes mayores a 15 años, obtenidas en el Centro Radiográfico CERPAX de la ciudad de Cochabamba (Bolivia) desde mayo de 2017 hasta diciembre de 2017. 
Todas las tomografías fueron capturadas por un equipo Planmeca Promax 3D Mid (Planmeca OY, Finlandia) con un tamaño de voxel isotróprico entre 0,15 y $0,20 \mathrm{~mm}$, y evaluadas por el software Romexis versión 3.6.0. Las imágenes fueron adquiridas con $90 \mathrm{kV}$ y $10 \mathrm{~mA}$, y un tiempo de exposición de entre 12 y 13 segundos. El grosor de corte fue de 0,2 $\mathrm{mm}$.

Fueron incluidas aquellas tomografías con un tamaño de voxel mínimo de 0,2 $\mathrm{mm}$ y que abarcaban desde la región del primer premolar superior derecho hasta el contralateral, y desde el piso de las fosas nasales hasta la cresta alveolar. Fueron excluidas aquellas tomografías con calidad de imagen insuficiente, con evidencia de algún procedimiento quirúrgico en la zona tales como implantes e injertos óseos, presencia de dientes retenidos, lesiones óseas, malformaciones, síndromes y traumatismos en la zona.

Las variables tomadas en cuenta fueron la presencia y cantidad de conductos accesorios menores a $1 \mathrm{~mm}$ de diámetro, la presencia y cantidad de conductos accesorios iguales o mayores a $1 \mathrm{~mm}$, el diámetro mayor de los conductos que tengan un diámetro mínimo de $1 \mathrm{~mm}$, la emergencia de los conductos en relación a las piezas dentarias o al foramen incisivo, la emergencia de los conductos a nivel de las tablas óseas, el estado dental de la zona donde se encuentran y la dirección o trayecto de los conductos en sentido cefálico.

Se realizó la calibración de la cursante de la especialidad. Se procedió a la selección de la muestra y la recolección de los datos por medio de fichas previamente diseñadas. Para esto, se posicionó el volumen en la ventana de reconstrucción multiplanar (MPR), de tal forma que se siga la dirección del paladar duro tanto en los cortes coronales como en los sagitales, donde se estableció la presencia 0 ausencia de conductos accesorios. La emergencia fue evaluada en los cortes sagitales y axiales, tanto a nivel de las piezas dentarias como de las tablas óseas y el estado dental del área; se posicionó el volumen de tal forma que en los cortes sagitales y coronales se siga la dirección del conducto donde se procedió a la medición del diámetro y verificó la dirección del conducto en sentido cefálico. Se realizaron varias mediciones del diámetro a diferentes niveles del conducto y fue registrada la mayor.

Los datos fueron registrados y procesados en el programa estadístico IBM SPSS Statistics versión 20.0.0.0.

La identidad de los pacientes es revelada en ningún momento. La base de datos utilizada incluyó tomografías obtenidas por diversos motivos, como implantes dentales, endodoncia o evaluación de dientes retenidos. Ningún paciente fue expuesto únicamente con motivo de investigación.

\section{RESULTADOS}

De los 300 casos evaluados se encontraron 93 pacientes $(31 \%)$ con conductos menores a $1 \mathrm{~mm}$ y 142 pacientes $(47,33 \%)$ con algún conducto con diámetro igual o mayor a $1 \mathrm{~mm}$ (figura 1), de los cuales, 83 son mujeres y 59 varones, esto mostró que si hay asociación estadísticamente significativa $(p=0,038$ con la prueba exacta de Fisher), lo que indica que los varones presentan más posibilidad de tener un conducto igual o mayor a $1 \mathrm{~mm}$ que las mujeres (tabla 1).

De los 300 casos evaluados hubo 101 personas $(33,67 \%)$ que presentaron un conducto, 36 presentaron (12\%) dos conductos, 4 presentaron 3 conductos y 1 paciente presentó 4 conductos (figura 2). En los 142 pacientes que presentaron algún conducto accesorio igual o mayor a $1 \mathrm{~mm}$ de diámetro, se encontraron 189 conductos. 


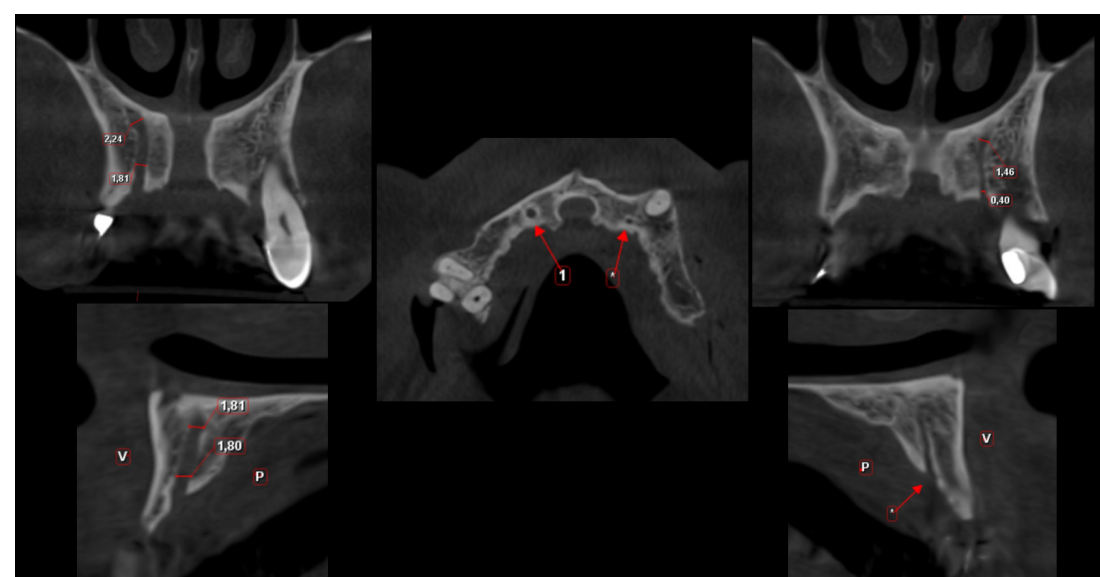

FIgURA 1. CoRTES DE TOMOGRAFíA COMPUTARIZADA DE HAZ CÓNICO QUE MUESTRAN DOS CONDUCTOS ACCESORIOS: EL PRIMERO CON UN DIÁMETRO DE 2,24 MM Y EL SEGUNDO MENOR A 1 MM.

Tabla 1. Presencia de conductos iguales o mayores a 1 mm, según sexo

\begin{tabular}{|l|c|c|c|c|}
\hline \multirow{2}{*}{ Sexo } & \multicolumn{3}{|c|}{ Conductos mayores a $1 \mathrm{~mm}$} \\
\cline { 2 - 5 } Femenino & $\mathrm{n}$ & Ausente & Presente & Total \\
\hline \multirow{3}{*}{ Masculino } & $\%$ & $56,77 \%$ & $43,23 \%$ & 192 \\
\hline & $\mathrm{n}$ & 49 & 59 & $100,00 \%$ \\
\hline Total & $\%$ & $45,37 \%$ & $54,63 \%$ & 108 \\
\hline & $\mathrm{n}$ & 158 & 142 & 300 \\
\hline
\end{tabular}

$p=0,038$

Prueba exacta de Fisher

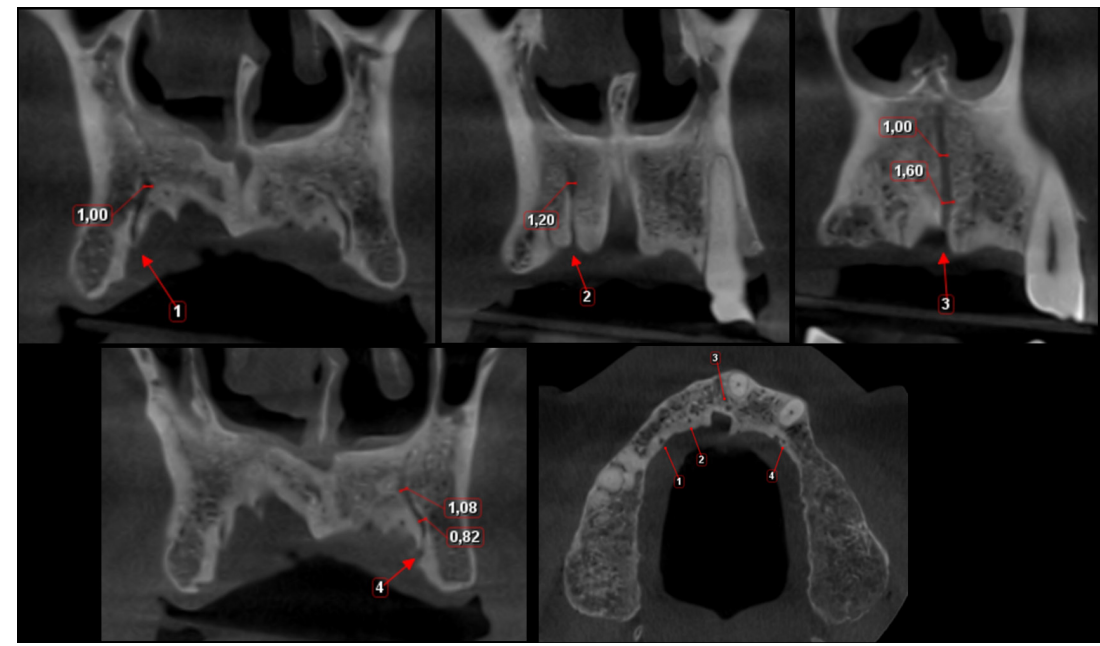

Figura 2. CoRTES TOMOGRÁFICOS PERTENECIENTES A UN PACIENTE DEL SEXO MASCULINO DE 60 AÑOS CON CUATRO CONDUCTOS ACCESORIOS IGUALES O MAYORES A $1 \mathrm{MM}$. 


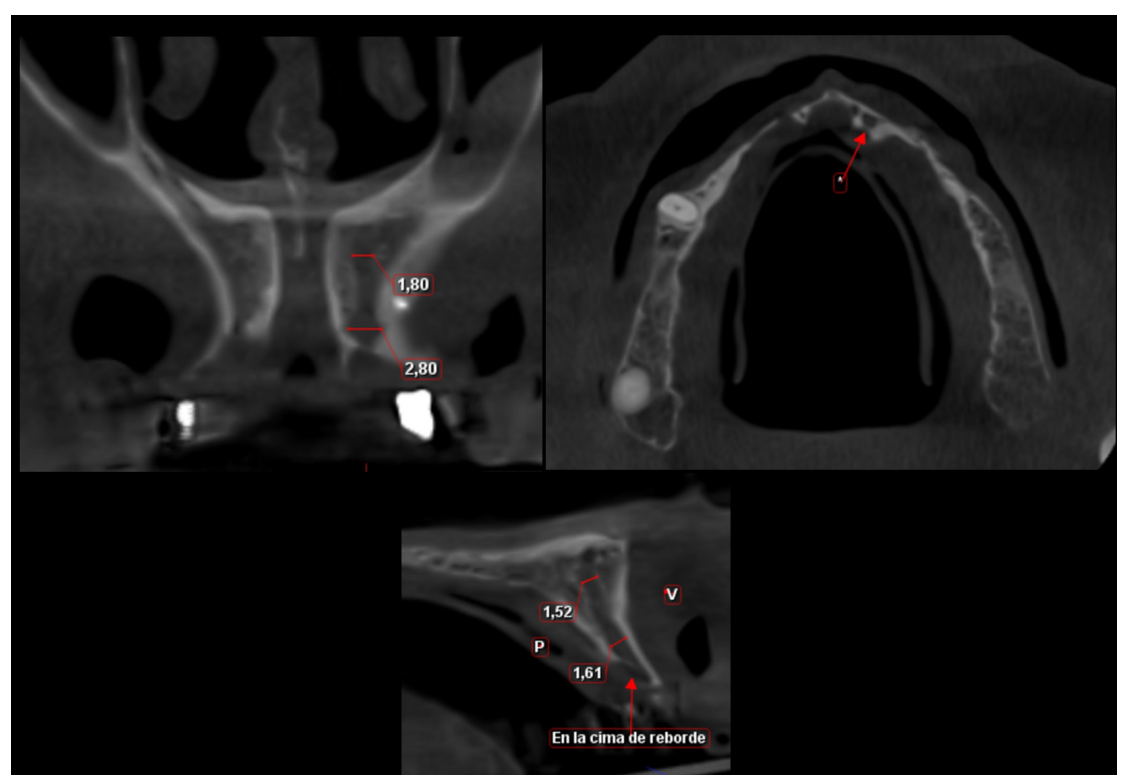

FIGURA 3. CORTES TOMOGRÁFICOS DE UN CONDUCTO ACCESORIO DE 2,80 MM DE DIÁMETRO CON EMERGENCIA EN LA CIMA DEL REBORDE ÓSEO ALVEOLAR EN UNA ZONA DESDENTADA.

TABLA 2. Asociación ENTRE EL DIÁmETRO dEL CONDUCtO Y EL ESTADO DENTAL DE LA ZONA DONDE SE ENCUENTRAN

\begin{tabular}{|l|c|c|c|c|}
\hline & Estado dental de la zona & N & Media & D. E. \\
\hline \multirow{2}{*}{ Diámetro del conducto accesorio } & Dentada & 116 & 1,37 & 0,28 \\
\hline & Desdentada & 73 & 1,55 & 0,47 \\
\hline
\end{tabular}

$p=0,001$

Prueba de T de Student entre el grupo dentado y desdentado

TABLA 3. TIPO DE EMERGENCIA DE LOS CONDUCTOS ACCESORIOS IGUALES O MAYORES A 1 MM CON RELACIÓN A LAS PIEZAS DENTARIAS O AL FORAMEN INCISIVO, SEGÚN SEXO

\begin{tabular}{|l|l|c|c|c|c|c|c|c|}
\hline \multicolumn{7}{|c|}{ Emergencia a nivel de piezas dentarias de los conductos accesorios } \\
\hline Sexo & $\begin{array}{c}\text { Región } \\
\text { de } \\
\text { incisivos } \\
\text { centrales }\end{array}$ & $\begin{array}{c}\text { Entre } \\
\text { incisivos } \\
\text { central y } \\
\text { lateral }\end{array}$ & $\begin{array}{c}\text { Región } \\
\text { de } \\
\text { incisivos } \\
\text { laterales }\end{array}$ & $\begin{array}{c}\text { Región } \\
\text { canina }\end{array}$ & $\begin{array}{c}\text { Anterior } \\
\text { al } \\
\text { foramen } \\
\text { incisivo }\end{array}$ & $\begin{array}{c}\text { Lateral al } \\
\text { foramen } \\
\text { incisivo }\end{array}$ & Total \\
\hline Femenino & $\mathrm{n}$ & 35 & 29 & 13 & 6 & 14 & 12 & 109 \\
\hline & $\%$ & $32,11 \%$ & $26,61 \%$ & $11,93 \%$ & $5,50 \%$ & $12,84 \%$ & $11,01 \%$ & $100,00 \%$ \\
\hline Masculino & $\mathrm{n}$ & 24 & 17 & 17 & 2 & 17 & 3 & 80 \\
\hline \multirow{2}{*}{ Total } & $\%$ & $30,00 \%$ & $21,25 \%$ & $21,25 \%$ & $2,50 \%$ & $21,25 \%$ & $3,75 \%$ & $100,00 \%$ \\
\hline & $\mathrm{n}$ & 59 & 46 & 30 & 8 & 31 & 15 & 189 \\
\hline
\end{tabular}

Prueba de Chi-cuadrado 


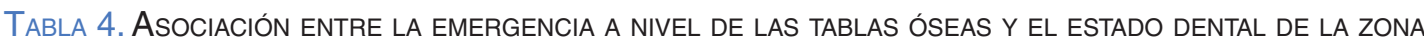
DONDE SE ENCUENTRAN

\begin{tabular}{|c|c|c|c|c|c|}
\hline & & & \multicolumn{2}{|c|}{ Estado dental de la zona } & \multirow[t]{2}{*}{ Total } \\
\hline & & & Dentada & Desdentada & \\
\hline \multirow{6}{*}{$\begin{array}{l}\text { Emergencia del conducto } \\
\text { accesorio a nivel de las } \\
\text { tablas óseas }\end{array}$} & \multirow{2}{*}{ Palatino } & $n$ & 112 & 42 & 154 \\
\hline & & $\%$ & $72,7 \%$ & $27,3 \%$ & $100,0 \%$ \\
\hline & \multirow{2}{*}{$\begin{array}{l}\text { En la cima del reborde } \\
\text { óseo alveolar }\end{array}$} & $n$ & 4 & 28 & 32 \\
\hline & & $\%$ & $12,5 \%$ & $87,5 \%$ & $100,0 \%$ \\
\hline & \multirow{2}{*}{ Vestibular } & $n$ & 0 & 3 & 3 \\
\hline & & $\%$ & $0,0 \%$ & $100,0 \%$ & $100,0 \%$ \\
\hline \multirow{2}{*}{\multicolumn{2}{|c|}{ Total }} & $n$ & 116 & 73 & 189 \\
\hline & & $\%$ & $61,4 \%$ & $38,6 \%$ & $100,0 \%$ \\
\hline
\end{tabular}

$\mathrm{p}<0,001$

Prueba de Chi cuadrado

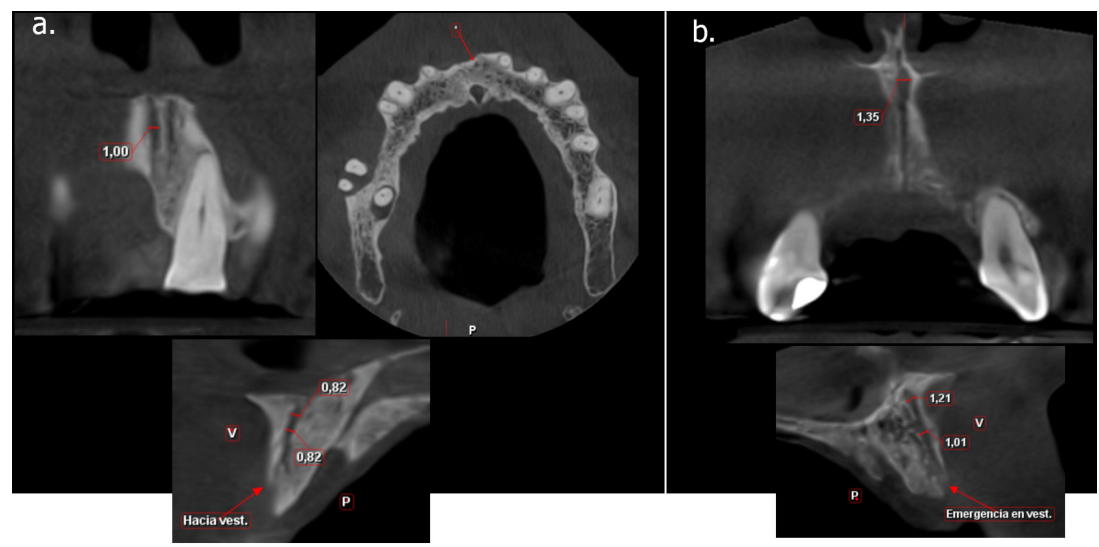

Figura 4. CoRTES TOMOGRÁFICOS DE dOS CASOS EN LOS QUE LOS CONDUCTOS ACCESORIOS EMERGEN A NIVEL DE LA TABLA ÓSEA VESTIBULAR EN ZONAS DESDENTADAS.

El diámetro medio de los 189 conductos es de $1,44 \mathrm{~mm}$. El diámetro mayor encontrado es de 2,80 $\mathrm{mm}$ (figura 3). El diámetro medio de los conductos en zonas dentadas es de $1,37 \mathrm{~mm}$ y en zona desdentadas es de $1,55 \mathrm{~mm}$, y se encontraron diferencias estadísticamente significativas entre ambos grupos ( $p=0,001$ prueba $T$ de Student), lo cual indica que hay un aumento del diámetro de los conductos en zonas desdentadas (tabla 2).

En la tabla 3 se observa la emergencia de los conductos con relación a las piezas dentarias $o$ al foramen incisivo. De los 189 conductos evaluados, 59 (31,2\%) emergen en la región de los incisivos centrales y $46(24,3 \%)$ entre los incisivos central y lateral. Del total, 31 conductos emergen de la región anterior al foramen incisivo y 30 en la región de los incisivos laterales. No se encontró ningún conducto cuya emergencia se produzca en la región posterior del foramen incisivo ni en la región de los premolares.

En la tabla 4 se evalúa la emergencia de los 189 conductos a nivel de las tablas óseas. Se encontró que 154 conductos emergen a nivel de la tabla ósea palatina, 32 en la cima del reborde óseo alveolar y 3 en la tabla ósea vestibular (figura 4). De los 154 conductos que emergen 
TABLA 5. DEscRipción dE LA DIRECCIÓN DE LOS CONDUCTOS ACCESORIOS

\begin{tabular}{|l|c|c|}
\hline & Conductos $(n)$ & Porcentaje (\%) \\
\hline Hacia el canalis sinuosus & 132 & $69,8 \%$ \\
\hline Hacia el piso de las fosas nasales & 24 & $12,7 \%$ \\
\hline No definida & 33 & $17,5 \%$ \\
\hline Total & 189 & $100,0 \%$ \\
\hline
\end{tabular}

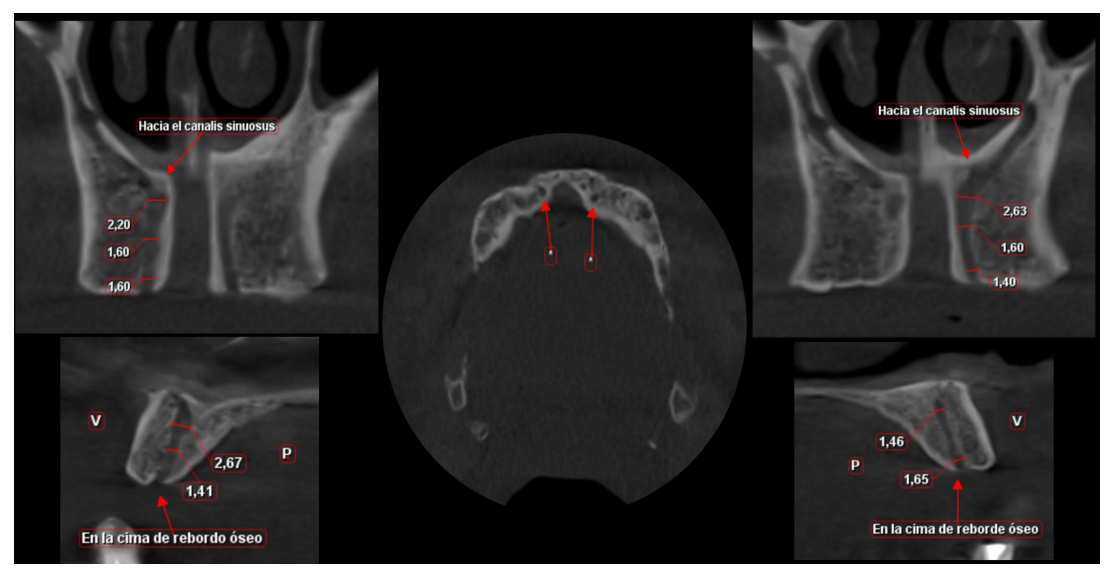

FiguRA 5. CoRTES TOMOGRÁFICOS DE DOS CONDUCTOS ACCESORIOS (2,67 Y 2,63 MM DE DIÁMETRO) CON EMERGENCIAS A NIVEL DE LOS INCISIVOS CENTRALES Y EN LA CIMA DEL REBORDE ÓSEO ALVEOLAR DE ZONAS DESDENTADAS, AMBOS CON DIRECCIÓN HACIA EL CANALIS SINUOSUS.

en la tabla ósea palatina, el $72,7 \%$ se encuentra en zonas dentadas; de los 32 conductos que emergen en la cima del reborde, el $87,5 \%$ se encuentra en zonas desdentadas, y los 3 conductos (100\%) que emergen en la tabla ósea vestibular se encuentran en zonas desdentadas. Existe una diferencia estadística significativa entre ambos $(p=0,00$ prueba de Chi-cuadrado), lo que indica que existe una tendencia de que la reabsorción del reborde óseo alveolar edéntulo condicione la emergencia de dichos conductos en la cima del reborde óseo o incluso en la tabla ósea vestibular.

En la tabla 5 se evalúa la dirección de los 189 conductos accesorios. El 69,8\% de los conductos tienen una dirección en sentido cefálico hacia el canalis sinuosus, pudiendo albergar al nervio alveolar anterior superior y la arteria alveolar anterior superior (figura 5). El 17,5\% no tienen una dirección definida. El 12,7\% tienen una dirección hacia el piso de las fosas nasales. No se encontró ningún conducto que tenga dirección hacia el conducto nasopalatino.

\section{DISCUSIÓN}

El $31 \%$ de los pacientes evaluados presenta algún conducto accesorio menor a $1 \mathrm{~mm}$. Este elevado porcentaje demuestra que existen conductos de calibre menor que no es posible observarlos por medio de técnicas bidimensionales y cuya relevancia clínica radica en el riesgo de lesionar el paquete vasculonervioso que albergan durante los procedimientos quirúrgicos de la región anterosuperior.

La frecuencia de conductos accesorios iguales o mayores a $1 \mathrm{~mm}$ encontrada en el presente estudio (47,33\%) es muy similar a las frecuencias encontradas 
en el estudio de Von Arx et al. (5) que reportaron un $55,1 \%$ de pacientes con al menos un canal accesorio. Machado et al (14) también encontraron una frecuencia de $51,7 \%$ de pacientes con algún conducto accesorio. Otros estudios encontraron frecuencias menores, Mañhaes et al. (9) reportaron una frecuencia de $36,20 \%$, pero no mencionan el diámetro de los conductos tomados en cuenta. Temmerman et al (23) hallaron una frecuencia del 32,9\%, tomando en cuenta únicamente la región canina. Sekerci et al. (4) con un $22,3 \%$ que pudiera deberse a que evalúa únicamente pacientes pediátricos. OliveiraSantos et al. (2) con $15,7 \%$, ya que solo toman en cuenta a los forámenes adicionales y no a los conductos, y Eshak et al. (7), un 13,1\% que se debe a que en su estudio consideran a todos los conductos accesorios de los maxilares, tanto superior como inferior. Estas variaciones entre los diferentes estudios también pueden ser por las diferentes características de las poblaciones estudiadas. La alta prevalencia de conductos accesorios encontrada en el presente estudio revela la importancia de evaluarlos cuando se consideren procedimientos quirúrgicos en la zona anterosuperior.

En el estudio se hallaron diferencias significativas en cuanto a la presencia de conductos iguales o mayores a 1 $\mathrm{mm}$ y el sexo, lo que demuestra que los varones tienen mayor tendencia a poseer conductos accesorios, esto va acorde con los estudios de Von Arx et al. (5) y Machado et al. (14) que encuentran una mayor prevalencia en hombres que en mujeres. Otros estudios no encontraron relación significativa.

Se encontró hasta 4 conductos accesorios en un solo paciente, razón por la cual la evaluación de las tomografías computarizadas del sector anterosuperior debe ser realizada de manera minuciosa.
El diámetro medio de los conductos en el presente estudio es de $1,44 \mathrm{~mm}$, lo que concuerda con estudios similares: Oliveira-Santos et al. (2), 1,4 mm; Gunler et al. (19), 1,37 mm; Von Arx et al. (5), 1,31 $\mathrm{mm}$; Temmerman et al. (23), 1,23 mm; Machado et al. (14), 1,19 mm; Sekerci et al. (4), 1,12 mm. Esto demuestra que el calibre del paquete vasculonervioso que albergan estos canales puede tener impacto clínico al ser lesionados. Tales daños van desde alteraciones neurológicas (parestesia, hiperestesia y dolor) hasta riesgo de hemorragia o pobre visualización del área quirúrgica ocasionado por el sangrado. El presente estudio no encontró diferencias significativas del diámetro entre hombres y mujeres, este resultado coincide con la mayoría, a excepción de Gunler et al. (19), quienes encontraron que el diámetro es mayor en pacientes varones.

El mayor diámetro reportado hasta la fecha es de 2,58 $\mathrm{mm}$ (14). El presente estudio encontró un diámetro máximo de $2,80 \mathrm{~mm}$, lo cual refuerza la importancia clínica al existir conductos de calibre clínicamente relevantes que no pueden ser pasados por alto en determinadas intervenciones quirúrgicas. Se demostró que en las zonas desdentadas se encontraron conductos accesorios de mayor diámetro que en las dentadas, por lo que los conductos tienden a aumentar su diámetro con la pérdida de las piezas dentarias, tal como puede ocurrir con el seno maxilar o el conducto nasopalatino (27).

La mayor emergencia de los conductos accesorios se da a nivel de los incisivos centrales, al igual que el estudio de Von Arx et al. (5). Los conductos emergentes a nivel del incisivo lateral ocupan la cuarta posición en el presente estudio, a diferencia de Sekerci et al. (4), Eshak et al. (7) y Gunler et al. (19), en los que la emergencia mayor se produce a este nivel. Esto demuestra que no solo 
debemos considerar al conducto nasopalatino en la zona de los incisivos, sino también a los conductos accesorios presentes.

Todos los estudios que toman en cuenta la emergencia a nivel de las tablas óseas concuerdan con el presente estudio en cuanto a la mayor emergencia a nivel palatino de las piezas dentarias (4, 5, 14, 19). No obstante, se demostró que en pacientes desdentados existe mayor tendencia a que dicha emergencia se localice en la cima del reborde óseo alveolar y todos los conductos que emergen a nivel de la tabla ósea vestibular se encuentran en zonas desdentadas, esto se explica por la reabsorción ósea alveolar que ocurre después de la pérdida de las piezas dentarias, lo cual genera que estructuras anatómicas importantes como el foramen mentoniano y el foramen incisivo emerjan en la cima del reborde óseo alveolar (28). Este aspecto es de gran relevancia, principalmente en el momento de la inserción de implantes dentales.

En el presente estudio, el 69,8\% de los conductos accesorios se dirigen hacia el CS, lo cual concuerda con el estudio de Von Arx et al. (5). Sin embargo, OliveiraSantos et al. (2) encontró mayor cantidad de conductos que se dirigen hacia el piso de las fosas nasales, al igual que Sekerci et al. (4). La mayor frecuencia de conductos que se dirigen hacia el CS revela que estos pueden contener en su interior al nervio alveolar anterior superior y a la arteria alveolar anterior. Aunque en el presente estudio no se encontraron conductos que se unan superiormente al conducto nasopalatino, Gunler et al. (19) reportó un caso; Sekerci et al. (4), 2 casos, y Oliveira-Santos et al. (2), otros 2 casos. Esto pudiera explicarse por las características poblacionales de las diferentes muestras estudiadas.
Se recomienda realizar más estudios en diferentes contextos poblacionales para evaluar posibles cambios de acuerdo a la población estudiada, así como evaluar la asociación entre diámetro de los conductos y emergencia con el estado dental de los pacientes en muestras mayores de población. Finalmente, se recomienda realizar investigaciones sobre el contenido real de los conductos accesorios.

\section{CONCLUSIONES}

El alto porcentaje de conductos iguales o mayores a $1 \mathrm{~mm}$, y el elevado número posible de conductos por paciente demuestran la necesidad de realizar una evaluación tomográfica previa a cualquier procedimiento quirúrgico en la región anterosuperior. La posibilidad de tener conductos accesorios de calibre significativo nos lleva a tomar precauciones para evitar lesiones en el paquete vasculonervioso, principalmente en pacientes sin dientes, ya que el diámetro de los conductos en áreas desdentadas tiende a ser mayor que en áreas dentadas.

Se debe realizar un adecuado diagnóstico de conductos accesorios en pacientes edéntulos que vayan a ser sometidos a cirugías de implantes e injertos óseos a fin de aumentar el éxito de los tratamientos y evitar daño de estructuras neurovasculares que puedan emerger en la cima del reborde óseo alveolar y en la tabla ósea vestibular.

El elevado porcentaje de conductos que se dirigen hacia el CS nos lleva a realizar una planificación adecuada de todo procedimiento quirúrgico para evitar daños al contenido de los conductos accesorios, que pueden derivar en alteraciones neurosensoriales.

Contribución del autor: Natalia Nilda Soto Terán ha participado en la 
concepción del artículo, la recolección de datos, su redacción y aprobación de la versión final.

Fuente de financiamiento: Autofinanciada.
Conflictos de interés: La autora declara no tener conflictos de interés de ningún tipo.

\section{REFERENCIAS BIBLIOGRÁFICAS}

1. Ozcakir-Tomruk C, Dolekoglu S, Oskurt-Kayahan Z, ilguy D. Evaluation of morphology of the nasopalatine canal using cone-beam computed tomography in a subgroup of Turkish adult population. Surg Radiol Anat. 2016; 38 (1): 65-70.

2. Olivera-Santos C, Rubira-Bullen IR, Monteiro SA, León JE, Jacobs R. Neurovascular anatomical variations in the anterior palate observed on CBCT images. Clin Oral Implants Res. 2013; 24 (9): 1044-1448.

3. Kose E, Sekerci AE, Soylu E, Nazlim S. An extremely rare anatomical variation bilateral canalis sinuosus and nasopalatine duct cyst and role of CBCT in diagnosis. Int J Sci Res. 2014; 3: 361-3.

4. Sekerci AE, Cantekin K, Aydinbelge M. Cone beam computed tomography analysis of neurovascular anatomical variations other than the nasopalatine canal in anterior maxilla in a pediatric population. Surg Radiol Anat. 2015; 37 (2): 181-6.

5. Von-Arx T, Lozanoff S, Sendi P, Bornstein MM. Assesment of bone channels other than the nasopalatine canal in the anterior maxilla using limited cone beam computed tomography. Surg Radiol Anat. 2013; 35 (9): 783-90.

6. Neves FS, Crusoé-Souza M, Franco LC, Caria PH, Bonfim-Almeida P, CrusoéRebello I. Canalis sinuosus: a rare anatomical variation. Surg Radiol Anat. 2012; 34 (6): 563-6.

7. Eshak M, Brooks S, Abdel-Wahed N, Edwards PC. Cone beam CT evaluation of presence of anatomic accessory canals in the jaws. Dentomaxillofac Radiol. 2014; 43: 1-8.

8. Wanzeler AM, Marinho CG, Alves-Junior SM, Manzi FR, Tufi FM. Anatomical study of the canalis sinuosus in 100 cone beam conputed tomography examinations. Oral Maxillofac Surg. 2015; 19 (1): 49-53.

9. Manhaes-Junior LR, Villaca-Carvalho MF, Moraes ME, Lopes SL, Silva MB, Junqueira JL. Location and classification of canalis sinuosus for cone beam computed tomography: avoiding misdiagnosis. Braz Oral Res. 2016; 30 (1): 1-8.

10. Torres MG, de-Faro-Valverde L, Vidal MT, Crusoé-Rebello IM. Branch of the canalis sinuosus: a rare anatomical variation-a case report. Surg Radiol Anat. 2015; 37 (7): 879-81. 
11. Kasahara N, Morita W, Tanaka R, Hayashi T, Kenmotsu S, Ohshima H. The relationships of the maxillary sinus with the superior alveolar nerves ans vessels as demonstrated by Cone-Beam CT combined with u-CT and histological analyses. The anatomical record. 2016; 299: 669-78.

12. Jones FW. The anterior superior alveolar nerve and vessels. J Anat. 1939; 73 (Pt 4): 583-591.

13. Von-Arx T, Lozanoff S. Anterior superior alveolar nerve (ASAN). Swiss Dent. J. 2015; 125 (11): 1202-9.

14. Machado VC, Chrcanovic BR, Felippe MB, Manhaes-Junior LR, de-Carvalho PS. Assessment of accesory canals of the canalis sinuosus: a study of 1000 cone beam computed tomography examinations. Int J. Oral Maxillofac. Surg. 2016; 45 (12): 1586-91.

15. Song WC, Kim JN, Yoo JY, Lee JY, Won SY, Hu KS, et al. Microanatomy of the infraorbital canal and its connecting canals in the maxilla using 3-D reconstruccion of microcomputed tomographic images. J Craniofac Surg. 2012; 23 (4): 11841887.

16. Olenczak JB, Hui-Chou HG, Aguila DJ, Shaeffer CA, Dellon AL, Manson PN. Posttraumatic midface pain: clinical significance of the anterior superior alveolar nerve and canalis sinuosus. Annals of Plastic Surgery. 2015; 75 (5): 543-7.

17. Mraiwa N, Jacobs R, Van-Cleynenbreugel J, Sanderink G, Schutyser F, Suetens $P$, et al. The nasopalatine canal revisted using 2D and 3D CT imaging. DentomaxiIlofac Radiol. 2004; 33: 396-402.

18. Jacobs R, Lambrichts I, Liang X, Martens W, Mraiwa N, Adriaensens P, et al. Neurovascularization of the anterior jaw bones revisted using high-resolution magnetic resonance imaging. Oral Surg Oral Med Pathol Oral Radiol Endod. 2007; 103 (5): 683-93.

19. Gunler G, Delilbasi C, Esen E, Aydin K, Sakul U. Evaluation of the morphology of the canalis sinuosus using cone-beam computed tomography in patients with maxillary impacted canines. Imaging Sci Dent. 2017; 47: 69-74.

20. Bornstein MM, Balsiger R, Sendi P, Von-Arx T. Morphology of the nasopalatine canal and dental implant surgery: a radiographic analysis of 100 consecutive patients using limited cone-beam computed tomography. Clin. Oral Impl. Res. 2011; 22: 295-301.

21. Liang X, Jacobs R, Martens W, Hu Y, Adriaenses P, Quirynen M, et al. Macro-and micro-anatomical histological and computed tomography scan characterization of the nasopalatine canal. J Clin Periodontol. 2009; 36: 598-603.

22. Shelley AM, Rushton VE, Horner K. Canalis sinuosus mimicking a periapical inflammatory lesion. Br Dent J. 1999; 186 (8): 378-9.

23. Temmerman A, Hertelé S, Teughels W, Dekeyser C, Jacobs R, Quirynen M. Are panoramic images reliable in planning sinus augmentation procedures? Clin Oral Implants Res. 2011; 22 (2): 189-94. 
24. Apostolakis D, Bissoon AK. Radiographic evaluation of the superior alveolar canal: measurements of its diameter and of its position in relation to the maxillary sinus floor: a cone beam computerized tomography study. Clin. Oral Implants. Res. 2014; 25 (5): 553-9.

25. Murakami G, Ohtsuka K, Sato I, Moriyama H, Shimada K, Tomita H. The superior alveolar nerves: their topographical relationship and distribution to the maxillary sinus in huma adults. Okajimas Folia Anat. Jpn. 1994; 70 (6): 319-28.

26. Kim J, Abdala-Junior R, Aoki E, Baladi M, Cortes A, Watanabe P, et al. Canalis Sinuosus and radiographic procedures in the region of anterior maxilla. Clin Lab Res Den. 2015; 21 (3): 180-4.

27. Mardinger O, Namani-Sadan N, Chaushu G, Schwarts-Arad D. Morphologic changes of the nasopalatine canal related to dental implantation: a radiologic study in different degrees of absorbed maxillae. J Periodontol. 2008; 79 (9): 165962.

28. Chrcanovic BR, Abreu MH, Custodio AL. Morphological variation in dentate and edentulous human mandibles. Surg Radiol Anat. 2011; 33: 203-13.

29. Hutchinson EF, Farella M, Kramer B. Importance of teeth in maintaining the morphology of the adult mandible in humans. Eur J Oral Sci. 2015; 123: 341-49.

30. Tozum TF, Guncu GN, Yildirim YD, Yilmaz HG, Galindo-Moreno P, Velasco-Torres $M$, et al. Evaluation of maxillary incisive canal characteristics related to dental implant treatment with computerized tomography: a clinical multicenter study. J Periodontol. 2012; 83 (3): 337-43. 